\title{
New directions in neighbourhood research-a commentary on Lovasi et al. (2016): Cause and context: place-based approaches to investigate how environments affect mental health
}

\author{
Peter Schofield $^{1} \cdot$ Jayati Das-Munshi $^{1}$
}

Received: 18 November 2016/Accepted: 15 December 2016/Published online: 4 January 2017

(C) Springer-Verlag Berlin Heidelberg 2017

We now live in a world where our sense of place is no longer fixed but increasingly fluid and subject to rapid change. Two large scale population changes in particular, urbanisation and migration, have become central to debates about how we live in the twenty-first century. Both have important mental health correlates and, as inherently spatial phenomena, both present a specific set of challenges for psychiatric epidemiology. Given the relevance of these topics it is not surprising that research into the effect of neighbourhood level factors on health has expanded dramatically in recent decades. One recent review of neighbourhood studies uncovered 1369 relevant papers published in journals linked to epidemiology in the 15 years since 1990 [1]. This is partly due to more data being available along with the widespread adoption of statistical methods, such as multilevel modelling, to facilitate analysis. However, despite the volume of published research, it is argued, few studies have been able to reach any definitive conclusions as to the relevance of neighborhood risk factors [1].

What we do know in psychiatric epidemiology is that rates of mental disorder, particularly psychosis, are raised in urban compared to rural areas. The adverse consequences of urban living are of course a long running theme in mental health research. For example, it was nearly 80 years ago that Faris and Dunham first looked at the effects of urban living on psychosis rates in Chicago [2].

This comment refers to the article available at doi:10.1007/s00127016-1300-x.

\footnotetext{
Peter Schofield

peter.1.schofield@kcl.ac.uk

King's College London, London, UK
}

This involved creating painstaking maps of the city and its suburbs showing associations between neighbourhood characteristics and rates of hospitalisation for different disorders. However, a long standing criticism of this and subsequent research has been that results could simply reflect reverse causality. It could be that living in an urban area is itself the result of a mental health problem, or underlying predisposition, rather than itself a causal factor. With cross-sectional study designs it is almost impossible to disentangle the two processes. This was one of the reasons that interest in the neighbourhood environment and mental health waned in the latter part of the twentieth century. However, in recent decades, thanks to a number of large scale cohort studies, it has been possible to disentangle the process of self-selection or 'social drift' into particular neighbourhood types to uncover relevant area level risk factors. We now know that rates of psychotic disorder are more than doubled when comparing urban and rural areas, and that there is a dose response relation between level of urbanicity and psychosis rates [3] with this confirmed by a range of large scale cohort studies in different international contexts [4]. Not only are these consistent with a causal effect but, because they concentrate on more severe mental illness, they cannot be easily explained by service use alone [3]. However, to date very little is known about what constituent elements of urban living contribute to this increased risk or the kind of interventions that might be required to tackle this.

A related field is migrant mental health. Again as a result of a number of large scale cohort studies there is now a large body of evidence to show that a range of ethnic minority/migrant groups are at increased risk of severe mental illness [5]. International comparison studies have shown this cannot be explained by any increased risk in the country of origin. That these elevated risks have been 
shown to persist from first to second generation migrants points to the relevance of the post migration environment. A particularly intriguing finding is the relation between neighbourhood ethnic density and rates of mental disorder, where higher own-ethnic group density appears to have a protective effect for different black and ethnic minority groups [6]. This runs counter to the usual notion that area deprivation-these are typically deprived urban areas-is the most relevant neighbourhood level factor. Again there is very little evidence about the mechanisms behind this or the implications for interventions.

So we have two examples of spatial disparities in rates of severe mental illness where, as yet, little is known about the underlying processes that could explain these differences. For some it is the absence of theory that has been the problem and, it has been argued, placing studies within a carefully formulated theoretical framework would help to uncover processes that have, so far, eluded more mechanistic research designs [7]. For others, this is primarily a methodological problem as, typically, studies continue to rely on cross-sectional data with often little attempt to address issues of causality in the design [1]. Instead, we risk adopting increasingly sophisticated statistical methods in an attempt to solve what are fundamentally study design problems.

Here, we have highlighted two broad areas where neighbourhood research is now at cross-roads. There has, of course, also been considerable interest in material factors, including the quality of the built environment and access to green spaces. However, their relevance to mental health is still unclear and it is difficult to determine exactly how any protective or adverse effects may operate. What partly drives these disparate areas of research is that results may ultimately inform relevant interventions. The effective assessment of urban mental health interventions is intrinsically appealing given the potential impact on the broader social determinants of health. For this reason we welcome the review by Lovasi et al. [8] for highlighting the importance of study design in combination with the application of novel statistical methods to this area of research. They discuss the use of local and large-scale interventions, with randomisation, to better understand causal inferences related to the effect of place on physical and mental health. Using examples from the US and China, they suggest opportunistically applying randomisation methods to policy interventions to make causal inferences. As they highlight, these present difficult ethical dilemmas, for example, where a desire to target interventions to the 'most needy areas' might compromise methodological rigour. Even if randomisation can be achieved, difficulties may still arise in determining causality, especially with outcomes which may take years to manifest, or if the nature of the 'exposure' remains unclear. Despite randomisation procedures holding the promise of analysis untainted by confounding, in reality this may be difficult to achieve, with individuals continuing to self-select into particular areas. Thoughtful application of statistical procedures such as propensity scores or adjustment through regression methods may help, but the issue of residual confounding and bias remains a concern [9].

Yet there is a growing need for robust evaluation of the impact of urban policies on mental health. Systematic reviews of urban regeneration policy and mental health have to date suggested only minor improvements in health as well as possible adverse-effects [10]. Large-scale urban regeneration programmes may bring immediate gains to local residents, in terms of cleaner and more aesthetically pleasing neighbourhoods [11], yet the long-term impact may not necessarily translate into better mental and physical health outcomes. This may be the case if long-term residents feel a sense of exclusion or if regeneration schemes have unintended consequences, for example, by disrupting local communities and social networks. As Lovasi and colleagues highlight, even with 'gold standard' methods, it may be difficult to disentangle which determinants in the social environment have led to improvements (or ill-effects) on the mental and physical health of residents.

Lovasi and colleagues further highlight the challenges of assessing mental health outcomes in the case studies they discuss. However, it is important to note that in some instances their concerns may be more specific to the US context (for example where studies rely on payment-based health systems to arrive at a proxy for mental health outcomes). Outside of the US, there are promising developments in the use of routine electronic health records for the assessment of mental health outcomes-especially with data collected on a whole population at country-level [3] or regional-level within cities [12]. These examples, incorporating adequate measures of relevant confounders exposures and outcomes, may hold the key to evaluating mental health outcomes following local or country-level policy interventions. Approaches which utilise readily available routine data may also mean that it is possible to adequately capture rapidly changing exposures. Despite guidance on the use of natural experiments [9], these approaches remain under-utilised and the suggestions by Lovasi and colleagues for novel methodological designs and techniques, including geographic and spatial modelling tools, are welcomed.

In conclusion, the review by Lovasi and colleagues draws our attention to a number of potential study designs and tools which could enhance our understanding of the impact of urban policy initiatives on mental and physical health. Yet methodological and conceptual challenges remain a vexing concern. Although it may be "time to 
expand the social epidemiological imagination" [1] the application of novel methods by epidemiologists to urban contexts demands caution, thought and care.

Acknowledgements Peter Schofield was funded as part of a UK Medical Research Council fellowship (grant no. MR/K021494/1) and Jayati Das-Munshi was funded by the Health Foundation working with the Academy of Medical Sciences.

\section{Compliance with ethical standards}

Conflict of interest The authors have no conflict of interest to report.

\section{References}

1. Oakes JM, Andrade KE, Biyoow IM, Cowan LT (2015) Twenty years of neighborhood effect research: an assessment. Curr Epidemiol Rep 2:80-87. doi:10.1007/s40471-015-0035-7

2. Faris R, Dunham W (1939) Mental disorders in urban areas: an ecological study of schizophrenia and other psychoses. The University of Chicago Press, Chicago

3. Pedersen CB (2001) Evidence of a dose-response relationship between urbanicity during upbringing and schizophrenia risk. Arch Gen Psychiatry 58:1039-1046

4. Vassos E, Pedersen CB, Murray RM et al (2012) Meta-analysis of the association of urbanicity with schizophrenia. Schizophr Bull 38:1118-1123. doi:10.1093/schbul/sbs096
5. Cantor-Graae E, Selten JP (2005) Schizophrenia and migration: a meta-analysis and review. Am J Psychiatry 162:12-24. doi:10. 1176/appi.ajp.162.1.12

6. Shaw RJ, Pickett KE (2011) The association between ethnic density and poor self-rated health among US Black and Hispanic people. Ethn Heal. doi:10.1080/13557858.2011.561428

7. March D, Hatch SL, Morgan C et al (2008) Psychosis and place. Epidemiol Rev 30:84-100

8. Lovasi GS, Mooney SJ, Muennig P, DiMaggio C (2016) Cause and context: place-based approaches to investigate how environments affect mental health. Soc Psychiatry Psychiatr Epidemiol 51(12):1571-1579

9. Craig P, Cooper C, Gunnell D et al (2011) Using natural experiments to evaluate population health interventions: guidance for producers and users of evidence. Med Res Counc 66(12):1-29

10. Thomson H, Atkinson R, Petticrew M, Kearns A (2006) Do urban regeneration programmes improve public health and reduce health inequalities? A synthesis of the evidence from UK policy and practice (1980-2004). J Epidemiol Community Health 60:108-115. doi:10.1136/jech.2005.038885

11. Thompson C, Lewis DJ, Greenhalgh T et al (2015) "Everyone was looking at you smiling": East London residents' experiences of the 2012 Olympics and its legacy on the social determinants of health. Heal Place 36:18-24. doi:10.1016/j.healthplace.2015.08. 008

12. Schofield P, Das-Munshi J, Mathur R et al (2016) Does depression diagnosis and antidepressant prescribing vary by location? Analysis of ethnic density associations using a large primary-care dataset. Psychol Med 46:1-9. doi:10.1017/S0033291715002913 